Supporting Information Binder, Crone, Haug, Menz, Kirsch

\title{
Direct Carbocyclization of Aldehydes with Alkynes: Combining Gold Catalysis with Aminocatalysis
}

\author{
Jörg T. Binder, Benedikt Crone, Timm T. Haug, Helge Menz, and Stefan F. Kirsch,* \\ Department Chemie, Technische Universität München, Lichtenbergstr. 4, D-85747 Garching, \\ Germany
}

\section{Supporting Information}

Representative experimental procedures for catalytic formation of $\mathbf{2}$ and $\mathbf{3}$, and copies of ${ }^{1} \mathrm{H}$ and ${ }^{13} \mathrm{C}$ NMR of compounds $\mathbf{3 a}-\mathbf{3 g}, \mathbf{2 h}-\mathbf{2 k}, \mathbf{5}, \mathbf{8}$, and $\mathbf{9}$.

General experimental details: All commercially available chemicals were used without further purification. ${ }^{1} \mathrm{H}$ NMR spectra were obtained on Bruker $500 \mathrm{MHz}$ FT-NMR, $360 \mathrm{MHz}$ FT-NMR and $250 \mathrm{MHz}$ FT-NMR spectrometers. ${ }^{13} \mathrm{C}$ NMR spectra were recorded at $90.6 \mathrm{MHz}$. Chemical shifts are reported in ppm relative to solvent signal. Multiplicity is indicated as follows: $\mathrm{s}$ (singlet); $\mathrm{d}$ (doublet); $\mathrm{t}$ (triplet); $\mathrm{m}$ (multiplet); dd (doublet of doublets). High resolution mass spectra and EI were determined on a Finnigan MAT 95S and MAT 8200. Flash chromatography was performed with E. Merck silica gel $(43-60 \mu \mathrm{m})$. The eluent used is reported in parentheses ( $\mathrm{P}=$ pentanes). Thin-layer chromatography (TLC) was performed on precoated glass-backed plates (Merck Kieselgel $60 \mathrm{~F}_{254}$ ), and components were visualized by observation under UV light or by treating the plates with $\mathrm{KMnO}_{4} / \mathrm{H}_{2} \mathrm{SO}_{4}$ followed by heating.

All formyl alkynes $\mathbf{1}$ were obtained following a general approached as described by others. ${ }^{1}$

\footnotetext{
${ }^{1}$ Buisine, O.; Aubert, C.; Malacria, M. Chem. Eur. J. 2001, 7, 3517.
} 


\section{General Procedures for the Cyclization Reactions}

General Procedure 1 (Synthesis of $\mathbf{2 h}$; Table 2, entry 7): A solution of $\mathbf{1 h}$ (100 $\mathrm{mg}, 0.42$ mmol) and $\left(c-\mathrm{C}_{6} \mathrm{H}_{11}\right)(i-\mathrm{Pr}) \mathrm{NH}(20 \mathrm{~mol} \%, 12 \mathrm{mg})$ in $\mathrm{CDCl}_{3}(0.4 \mathrm{~mL})$ was added to $\left[\left(\mathrm{Ph}_{3} \mathrm{PAu}\right)_{3} \mathrm{O}\right] \mathrm{BF}_{4}(7.5 \mathrm{~mol} \%, 45 \mathrm{mg})$, and the reaction vial was sealed, protected from light, and stirred at $70{ }^{\circ} \mathrm{C}$ for $18 \mathrm{~h}$ (until ${ }^{1} \mathrm{H}$ NMR analysis of the reaction mixture indicated complete conversion). The mixture was concentrated under reduced pressure. Purification of the residue by flash chromatography on silica gel (pentanes/EtOAc 95:5) gave $\mathbf{2 h}$ as a colorless oil (71mg, $0.30 \mathrm{mmol}, 71 \%) . R_{\mathrm{f}}=0.48$ (pentanes/ EtOAc 90:10).

General Procedure 2 (Synthesis of 3a; Table 1, entry 5): A solution of $\mathbf{1 a}(90 \mathrm{mg}, 0.40 \mathrm{mmol})$ and $(i-\mathrm{Pr})_{2} \mathrm{NH}\left(20 \mathrm{~mol} \%, 8 \mathrm{mg}\right.$; added as a $0.1 \mathrm{M}$ stock solution) in $\mathrm{CDCl}_{3}(0.4 \mathrm{~mL})$ was added to a mixture of $\operatorname{AgSbF}_{6}(11 \mathrm{mg}, 10 \mathrm{~mol} \%)$ and $\mathrm{PPh}_{3} \mathrm{AuCl}(16 \mathrm{mg}, 10 \mathrm{~mol} \%)$, and the reaction vial was sealed, protected from light, and stirred at $70{ }^{\circ} \mathrm{C}$ for $6 \mathrm{~h}$ (until TLC analysis of the reaction mixture indicated complete conversion). The mixture was concentrated under reduced pressure. Purification of the residue by flash chromatography on silica gel (pentanes/Et $\mathrm{E}_{2} \mathrm{O} 70: 30$ ) gave $\mathbf{3 a}$ as a colorless oil $(74 \mathrm{mg}, 0.33 \mathrm{mmol}, 82 \%) . R_{\mathrm{f}}=0.2$ (pentanes/ EtOAc 60:40).

\section{Compound Characterization Data}

\section{Dimethyl 3-formyl-4-methylcyclopent-3-ene-1,1-dicarboxylate (3a)}

${ }^{1} \mathrm{H}$ NMR $\left(360 \mathrm{MHz}, \mathrm{CDCl}_{3}\right): \delta=2.14(\mathrm{~s}, 3 \mathrm{H}), 3.23$ (s, $\left.4 \mathrm{H}\right), 3.74(\mathrm{~s}, 6 \mathrm{H}), 9.93(\mathrm{~s}, 1 \mathrm{H}) ;{ }^{13} \mathrm{C}$ NMR (90.6 MHz, $\left.\mathrm{CDCl}_{3}\right): \delta=14.0,38.8,47.8,53.2,56.8,135.2,157.5,171.9$, 187.2; LRMS (EI): 226 (35\%) $\left[\mathrm{M}^{+}\right], 195$ (46\%), 168 (100\%), 135 (93\%), 107 (98\%), 79 (76\%); HRMS $226.0840\left[226.0841\right.$ calcd for $\left.\mathrm{C}_{11} \mathrm{H}_{14} \mathrm{O}_{5}\left(\mathrm{M}^{+}\right)\right]$.

\section{4,4-Bis((tert-butyldiphenylsilyloxy)methyl)-2-methylcyclopent-1-enecarbaldehyde (3b)}

${ }^{1} \mathrm{H}$ NMR $\left(360 \mathrm{MHz}, \mathrm{CDCl}_{3}\right): \delta=1.03(\mathrm{~s}, 18 \mathrm{H}), 2.00(\mathrm{~s}, 3 \mathrm{H}), 2.42(\mathrm{~s}, 2 \mathrm{H}), 2.45(\mathrm{~s}, 2 \mathrm{H})$, 3.58-3.66 (m, 4 H), 7.20-7.36 (m, 8 H), 7.38-7.44 (m, 4 H), 7.59-7.66 (m, 8 H), 9.91 (s, 1 $\mathrm{H}) ;{ }^{13} \mathrm{C} \mathrm{NMR}\left(90.6 \mathrm{MHz}, \mathrm{CDCl}_{3}\right): \delta=14.4,19.5,27.1,36.1,46.0,47.7,66.8,127.8,129.8$, 133.6, 133.7, 135.8, 136.9, 160.8, 188.2; LRMS (EI): $589(50 \%)\left[\mathrm{M}^{+}-\mathrm{C}_{4} \mathrm{H}_{9}\right], 333(32 \%), 257$ (21\%), 197 (37\%), 135 (100\%), 91 (28\%); HRMS 589.2600 [589.2594 calcd for $\mathrm{C}_{37} \mathrm{H}_{41} \mathrm{O}_{3} \mathrm{Si}$ $\left.\left(\mathrm{M}^{+}-\mathrm{C}_{4} \mathrm{H}_{9}\right)\right]$. 


\section{4,4-Bis(methoxymethyl)-2-methylcyclopent-1-enecarbaldehyde (3c)}

${ }^{1} \mathrm{H} \mathrm{NMR}\left(360 \mathrm{MHz}, \mathrm{CDCl}_{3}\right): \delta=2.10(\mathrm{~s}, 3 \mathrm{H}), 2.41-2.44(\mathrm{~m}, 2 \mathrm{H}), 2.47-2.51(\mathrm{~m}, 2 \mathrm{H})$, 3.22-3.29 (m, $4 \mathrm{H}), 3.30(\mathrm{~s}, 6 \mathrm{H}), 9.96(\mathrm{~s}, 1 \mathrm{H}) ;{ }^{13} \mathrm{C} \mathrm{NMR}\left(90.6 \mathrm{MHz}, \mathrm{CDCl}_{3}\right): \delta=14.4,36.8$,

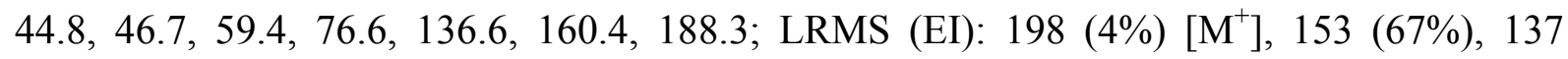
(25\%), 121 (47\%), 45 (100\%); HRMS 198.1257 [198.1256 calcd for $\mathrm{C}_{11} \mathrm{H}_{18} \mathrm{O}_{3}\left(\mathrm{M}^{+}\right)$].

\section{4,4-Bis(benzyloxymethyl)-2-methylcyclopent-1-enecarbaldehyde (3d)}

${ }^{1} \mathrm{H}$ NMR (360 MHz, $\left.\mathrm{CDCl}_{3}\right): \delta=2.09$ (s, $\left.3 \mathrm{H}\right), 2.50$ (s, $\left.2 \mathrm{H}\right), 2.53$ (s, $\left.2 \mathrm{H}\right), 3.37-3.44$ (m, 4 $\mathrm{H}), 4.51(\mathrm{~s}, 4 \mathrm{H}), 7.24-7.36(\mathrm{~m}, 10 \mathrm{H}), 9.96(\mathrm{~s}, 1 \mathrm{H}) ;{ }^{13} \mathrm{C} \mathrm{NMR}\left(90.6 \mathrm{MHz}, \mathrm{CDCl}_{3}\right): \delta=14.4$, 36.9, 45.0, 46.9, 73.4, 73.8, 127.6, 127.6, 128.4, 136.7, 138.7, 160.5, 188.3; LRMS (EI): 350 $(6 \%)\left[\mathrm{M}^{+}\right], 259$ (10\%), 229 (23\%), 121 (15\%), 91 (100\%); HRMS 350.1884 [350.1882 calcd for $\left.\mathrm{C}_{23} \mathrm{H}_{26} \mathrm{O}_{3}\left(\mathrm{M}^{+}\right)\right]$.

\section{4,4-Diacetyl-2-methylcyclopent-1-enecarbaldehyde (3e)}

${ }^{1} \mathrm{H} \mathrm{NMR}\left(360 \mathrm{MHz}, \mathrm{CDCl}_{3}\right): \delta=2.12$ (s, $\left.9 \mathrm{H}\right), 3.10$ (s, $\left.4 \mathrm{H}\right), 9.87$ (s, $\left.1 \mathrm{H}\right) ;{ }^{13} \mathrm{C} \mathrm{NMR}(90.6$ $\mathrm{MHz}, \mathrm{CDCl}_{3}$ ): $\delta=14.1,26.4,35.4,44.4,71.2,134.9,157.7,187.3,203.6$; LRMS (EI): 151 (50\%) $\left[\mathrm{M}^{+}-\mathrm{C}_{2} \mathrm{H}_{3} \mathrm{O}\right], 123$ (11\%), 109 (21\%), 81 (26\%), 43 (100\%); HRMS 151.0758 [151.0759 calcd for $\left.\mathrm{C}_{9} \mathrm{H}_{11} \mathrm{O}_{2}\left(\mathrm{M}^{+}-\mathrm{C}_{2} \mathrm{H}_{3} \mathrm{O}\right)\right]$.

\section{Ethyl 1-cyano-3-formyl-4-methylcyclopent-3-enecarboxylate (3f)}

${ }^{1} \mathrm{H}$ NMR (360 MHz, $\left.\mathrm{CDCl}_{3}\right): \delta=1.34$ (t, $\left.J=7.2 \mathrm{~Hz}, 3 \mathrm{H}\right), 2.19$ (s, $\left.3 \mathrm{H}\right), 3.14-3.44(\mathrm{~m}, 4 \mathrm{H})$, 4.29 (q, $J=7.2 \mathrm{~Hz}, 2 \mathrm{H}), 9.95$ (s, $1 \mathrm{H}) ;{ }^{13} \mathrm{C} \mathrm{NMR}\left(90.6 \mathrm{MHz}, \mathrm{CDCl}_{3}\right.$ ): $\delta=14.0,14.1,42.2$,

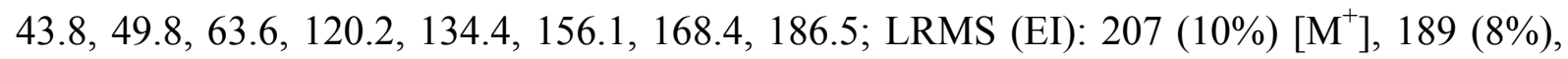
134 (100\%), 106 (36\%), 79 (27\%); HRMS 207.0897 [207.0895 calcd for $\mathrm{C}_{11} \mathrm{H}_{13} \mathrm{NO}_{3}\left(\mathrm{M}^{+}\right)$].

\section{3-Methyl-1H-indene-2-carbaldehyde (3g)}

${ }^{1} \mathrm{H}$ NMR $\left(360 \mathrm{MHz}, \mathrm{CDCl}_{3}\right): \delta=1.57(\mathrm{t}, J=2.3 \mathrm{~Hz}, 3 \mathrm{H}), 3.64(\mathrm{~d}, J=2.3 \mathrm{~Hz}, 2 \mathrm{H})$, 7.38-7.45 (m, 2 H), 7.52-7.55 (m, $1 \mathrm{H}), 7.58-7.60$ (m, $1 \mathrm{H}), 10.24$ (s, $1 \mathrm{H}) ;{ }^{13} \mathrm{C}$ NMR $(90.6$ $\left.\mathrm{MHz}, \mathrm{CDCl}_{3}\right): \delta=10.9,35.9,121.8,124.7,127.1,129.3,139.7,144.5,144.9,156.0,187.3$; LRMS (EI): 172 (52\%) [M+], 143 (74\%), 128 (100\%), 115 (30\%); LRMS (EI): 158 (77\%) $\left[\mathrm{M}^{+}\right], 130$ (73\%), $129(100 \%), 115$ (60\%),; HRMS: 158.0731 [158.0732 calcd for $\mathrm{C}_{11} \mathrm{H}_{10} \mathrm{O}$ $\left.\left(\mathrm{M}^{+}\right)\right]$. 


\section{Dimethyl 3-formyl-4-methylenecyclopentane-1,1-dicarboxylate (2h)}

${ }^{1} \mathrm{H}$ NMR $\left(250 \mathrm{MHz}, \mathrm{CDCl}_{3}\right): \delta=1.26(\mathrm{~s}, 3 \mathrm{H}), 2.23(\mathrm{~d}, J=14.0 \mathrm{~Hz}, 1 \mathrm{H}), 2.92-3.07(\mathrm{~m}, 3$ H), 3.73 (s, $3 \mathrm{H}$ ), 3.74 (s, $3 \mathrm{H}$ ), 4.95 (app. t, $J=2.2 \mathrm{~Hz}, 1 \mathrm{H}$ ), 5.22 (app. t, $J=1.9 \mathrm{~Hz}, 1 \mathrm{H}$ ), $9.29(\mathrm{~s}, 1 \mathrm{H}) ;{ }^{13} \mathrm{C}$ NMR $\left(90.6 \mathrm{MHz}, \mathrm{CDCl}_{3}\right): \delta=21.8,40.5,41.2,53.1,53.1,56.9,58.1$,

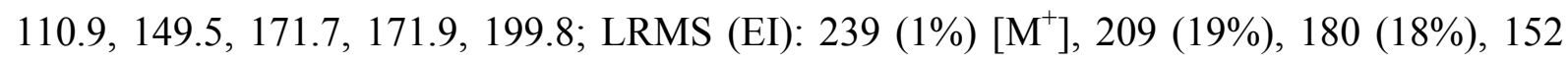
(95\%), 93 (100\%); HRMS 209.0815 [209.0814 calcd for $\left.\mathrm{C}_{11} \mathrm{H}_{13} \mathrm{O}_{4}\left(\mathrm{M}^{+}-\mathrm{CH}_{3} \mathrm{O}\right)\right]$.

\section{4,4-Bis(methoxymethyl)-2-methylenecyclopentanecarbaldehyde (2i)}

${ }^{1} \mathrm{H}$ NMR (250 MHz, $\left.\mathrm{CDCl}_{3}\right): \delta=1.25(\mathrm{~s}, 3 \mathrm{H}), 1.46(\mathrm{~d}, J=14.2 \mathrm{~Hz}, 1 \mathrm{H}), 2.18-2.41(\mathrm{~m}, 3$ H), $3.22-3.24(\mathrm{~m}, 4 \mathrm{H}), 3.32(\mathrm{~s}, 3 \mathrm{H}), 3.34$ (s, $3 \mathrm{H}), 4.87$ (app. t, $J=2.1 \mathrm{~Hz}, 1 \mathrm{H}), 5.12$ (app. t, $J=2.0 \mathrm{~Hz}, 1 \mathrm{H}), 9.32(\mathrm{~s}, 1 \mathrm{H}) ;{ }^{13} \mathrm{C} \mathrm{NMR}\left(90.6 \mathrm{MHz}, \mathrm{CDCl}_{3}\right): \delta=22.5,39.0,40.3$,

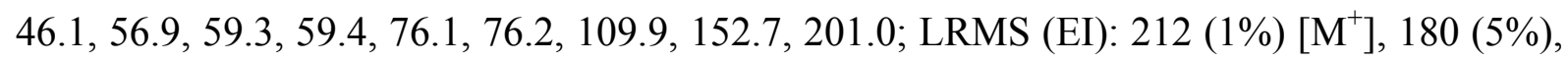
$180(18 \%), 152(8 \%), 119(50 \%), 107$ (100\%).

\section{4,4-Bis(benzyloxymethyl)-2-methylenecyclopentanecarbaldehyde (2j)}

${ }^{1} \mathrm{H}$ NMR (250 MHz, $\left.\mathrm{CDCl}_{3}\right): \delta=1.22(\mathrm{~s}, 3 \mathrm{H}), 1.53$ (d, $\left.J=14.1 \mathrm{~Hz}, 1 \mathrm{H}\right), 2.25-2.48$ (m, 3 H), 3.39 (m, 4 H), 4.50 (s, 2 H), 4.51 (s, 2 H), 4.85 (app. t, $J=2.1 \mathrm{~Hz}, 1 \mathrm{H}), 5.10$ (app. t, $J=$ $1.9 \mathrm{~Hz}, 1 \mathrm{H}), 7.27-7.37$ (m, $10 \mathrm{H}), 9.29$ (s, $1 \mathrm{H}) ;{ }^{13} \mathrm{C} \mathrm{NMR}\left(90.6 \mathrm{MHz}, \mathrm{CDCl}_{3}\right): \delta=22.5$, 39.1, 40.5, 46.3, 56.9, 73.3, 73.3, 73.4, 73.6, 109.9, 127.5, 127.6, 128.4, 138.8, 152.7, 200.9; LRMS (EI): 364 (1\%) [M+], 273 (10\%), 107 (35\%), 91 (100\%); HRMS 273.1489 [273.1491 calcd for $\left.\mathrm{C}_{17} \mathrm{H}_{21} \mathrm{O}_{3}\left(\mathrm{M}^{+}-\mathrm{C}_{7} \mathrm{H}_{7}\right)\right]$.

\section{2,3-Dihydro-2-methyl-1-methylene-1 $H$-indene-2-carbaldehyde (2k)}

${ }^{1} \mathrm{H}$ NMR $\left(360 \mathrm{MHz}, \mathrm{CDCl}_{3}\right): \delta=1.42(\mathrm{~s}, 3 \mathrm{H}), 2.82(\mathrm{~d}, J=16.8 \mathrm{~Hz}, 1 \mathrm{H}) 3.53(\mathrm{~d}, J=16.8$ $\mathrm{Hz}, 1 \mathrm{H}), 5.03$ (s, $1 \mathrm{H}), 5.71(\mathrm{~s}, 1 \mathrm{H})$ 7.20-7.29 (m, 3 H), 7.46-7.50 (m, $1 \mathrm{H}), 9.41$ (s, $1 \mathrm{H})$; ${ }^{13} \mathrm{C} \mathrm{NMR}\left(90.6 \mathrm{MHz}, \mathrm{CDCl}_{3}\right): \delta=10.9,35.9,121.8,124.7,127.1,129.3,139.7,144.5,144.9$, 156.0, 187.3; 172 (52\%) $\left[\mathrm{M}^{+}\right], 143$ (74\%), 128 (100\%), 115 (30\%); HRMS: 172.0889 [172.0888 calcd for $\left.\mathrm{C}_{12} \mathrm{H}_{12} \mathrm{O}\left(\mathrm{M}^{+}\right)\right]$.

\section{Dimethyl 5,6,7,7a-tetrahydro-3-methyl-4-oxo-2H-indene-1,1(4H)-dicarboxylate (5)}

${ }^{1} \mathrm{H}$ NMR $\left(360 \mathrm{MHz}, \mathrm{CDCl}_{3}\right): \delta=1.11-1.22(\mathrm{~m}, 1 \mathrm{H}), 1.70-1.83(\mathrm{~m}, 1 \mathrm{H}), 1.99-2.23(\mathrm{~m}, 6$ H), 2.42-2.48 (m, $1 \mathrm{H}), 2.77(\mathrm{~d}, J=18.4 \mathrm{~Hz}, 1 \mathrm{H}), 3.10(\mathrm{~d}, J=18.4 \mathrm{~Hz}, 1 \mathrm{H}), 3.68-3.71(\mathrm{~m}$, $1 \mathrm{H}), 3.72(\mathrm{~s}, 3 \mathrm{H}), 3.75(\mathrm{~s}, 3 \mathrm{H}) ;{ }^{13} \mathrm{C} \mathrm{NMR}\left(90.6 \mathrm{MHz}, \mathrm{CDCl}_{3}\right): \delta=15.9,23.6,27.7,40.8$,

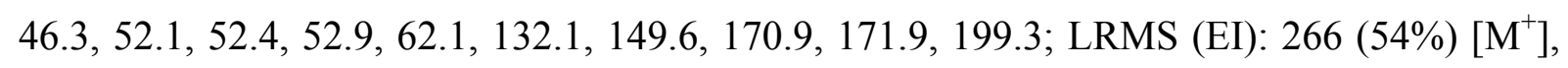
206 (70\%), 147 (100\%), 119 (26\%); HRMS: 266.1153 [266.1154 calcd for $\mathrm{C}_{14} \mathrm{H}_{18} \mathrm{O}_{5}\left(\mathrm{M}^{+}\right)$]. 


\section{Dimethyl 4,5,6,6a-tetrahydro-3-methyl-4-oxopentalene-1,1(3a $H)$-dicarboxylate (8)}

${ }^{1} \mathrm{H}$ NMR (360 MHz, $\left.\mathrm{CDCl}_{3}\right): 1.46-1.55(\mathrm{~m}, 1 \mathrm{H}), 1.77-1.78(\mathrm{~m}, 3 \mathrm{H}), 197-2.07(\mathrm{~m}, 1 \mathrm{H})$, 2.18-2.24 (m, $2 \mathrm{H}), 3.27-3.29(\mathrm{~m}, 1 \mathrm{H}), 3.58-3.65(\mathrm{~m}, 1 \mathrm{H}), 3.67(\mathrm{~s}, 3 \mathrm{H}), 3.72(\mathrm{~s}, 3 \mathrm{H})$, 5.49-5.51 (m, 1H); ${ }^{13} \mathrm{C}$ NMR (90.6 MHz, $\left.\mathrm{CDCl}_{3}\right): \delta=14.7,24.0,38.6,45.1,52.5,53.0$,

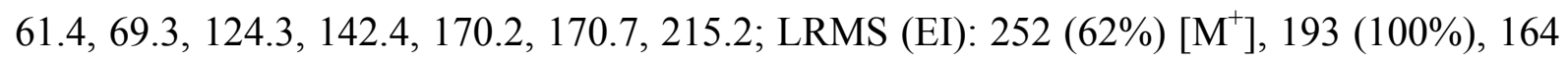
(62\%), 105 (60\%); HRMS: 252.0998 [252.0999 calcd for $\left.\mathrm{C}_{13} \mathrm{H}_{16} \mathrm{O}_{5}\left(\mathrm{M}^{+}\right)\right]$.

\section{5-Methyl-2-oxa-tricyclo[5.3.1.0 $\left.{ }^{1,5}\right]$ undec-3-ene-6,6-dicarboxylicacid dimethyl ester (9)}

${ }^{1} \mathrm{H}$ NMR $\left(360 \mathrm{MHz}, \mathrm{CDCl}_{3}\right): \delta=1.32-1.40(\mathrm{~m}, 1 \mathrm{H}), 1.41-1.51(\mathrm{~m}, 1 \mathrm{H}), 1.53-1.63(\mathrm{~m}, 2$ H), $1.65-1.74(\mathrm{~m}, 1 \mathrm{H}), 1.80-1.86(\mathrm{~m}, 1 \mathrm{H}), 1.91-1.99(\mathrm{~m}, 1 \mathrm{H}), 2.36-2.41(\mathrm{~m}, 1 \mathrm{H})$, 2.79-2.82 (m, $1 \mathrm{H}), 3.71(\mathrm{~s}, 3 \mathrm{H}), 3.74(\mathrm{~s}, 3 \mathrm{H}), 4.01-4.03(\mathrm{~m}, 1 \mathrm{H}), 4.73(\mathrm{t}, J=2.3 \mathrm{~Hz}, 1 \mathrm{H})$, $6.21(\mathrm{t}, J=2.3 \mathrm{~Hz}, 1 \mathrm{H}) ;{ }^{13} \mathrm{C} \mathrm{NMR}\left(90.6 \mathrm{MHz}, \mathrm{CDCl}_{3}\right): \delta=19.9,27.7,34.6,42.7,43.4,52.2$,

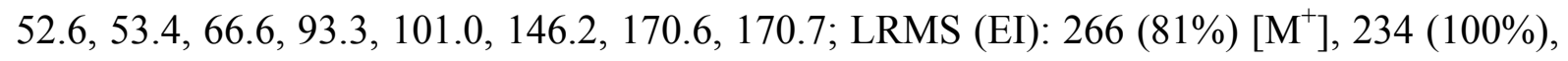
206 (95\%), 134 (82\%); HRMS: 266.1156 [266.1154 calcd for $\left.\mathrm{C}_{14} \mathrm{H}_{18} \mathrm{O}_{5}\left(\mathrm{M}^{+}\right)\right]$.

Key COSY, HMBC, and NOESY correlations of 9 are given in the illustration:
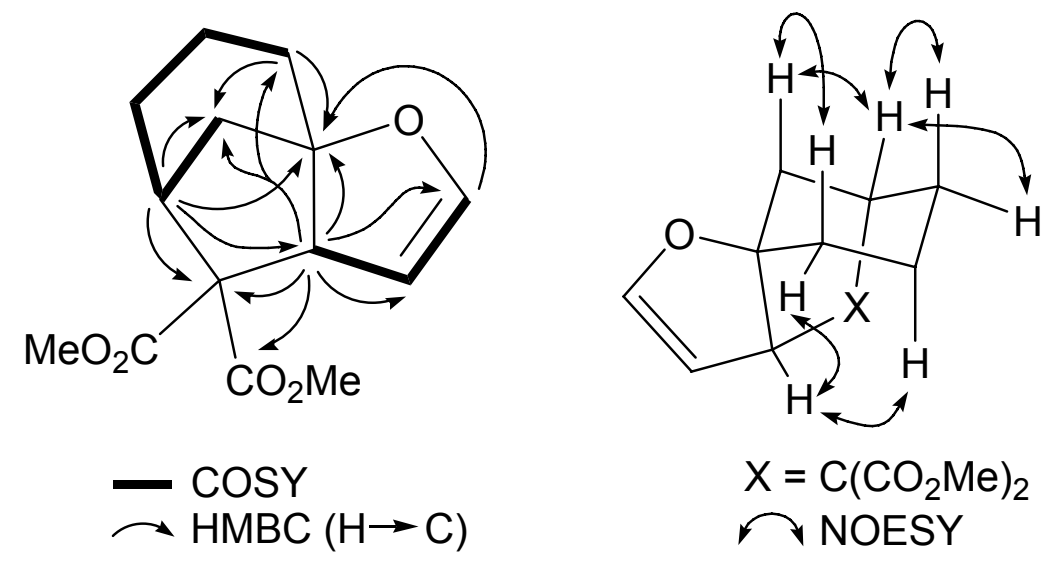


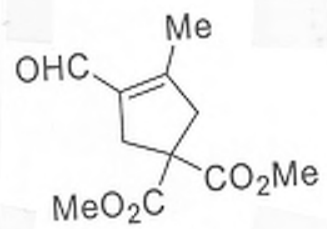

$3 a$

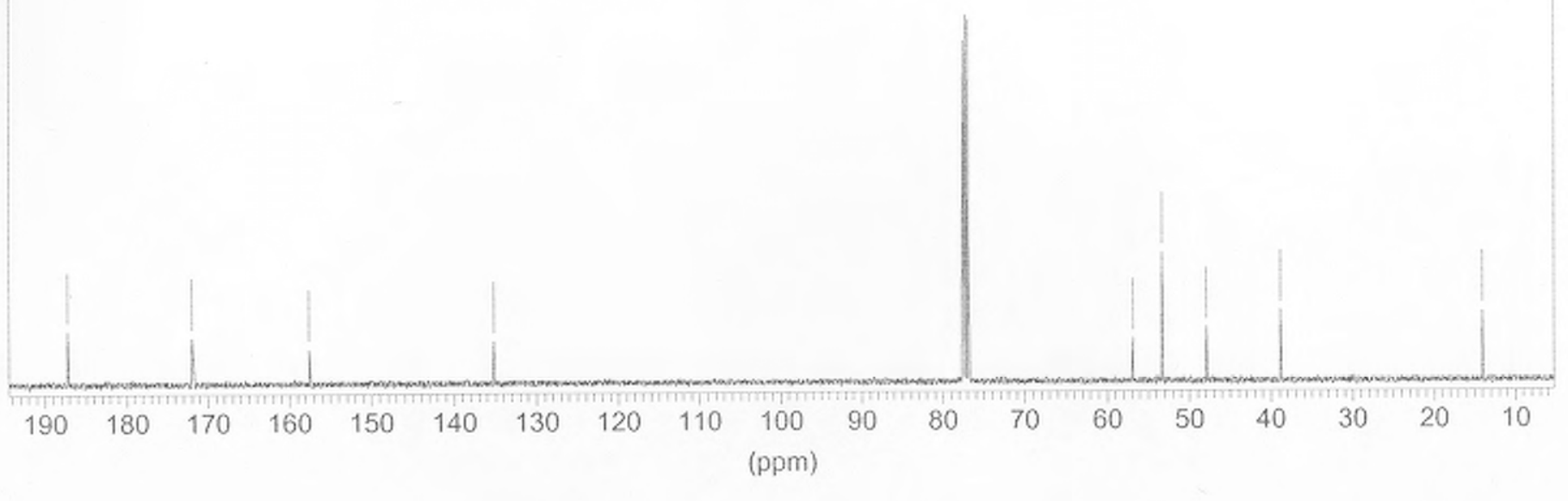



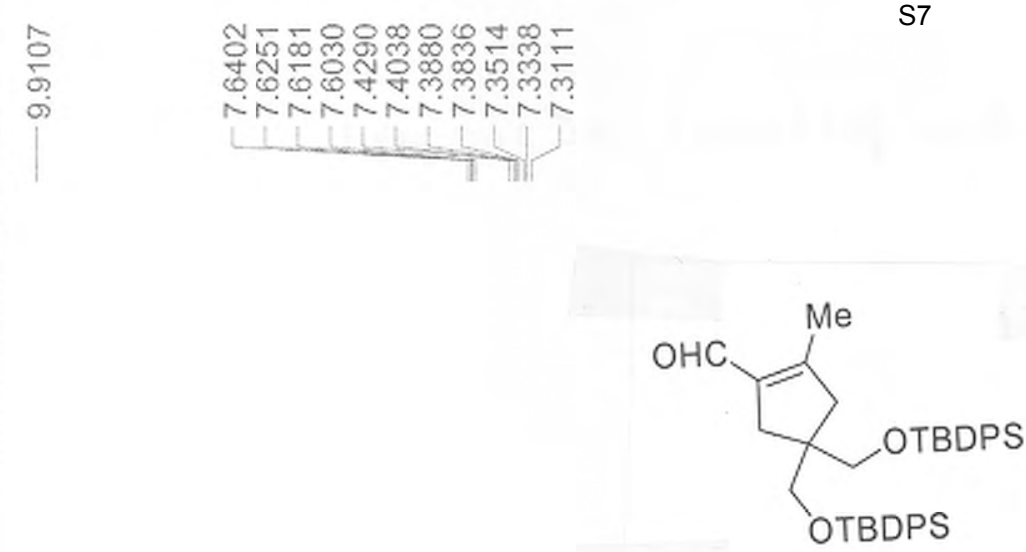

$3 \mathbf{b}$
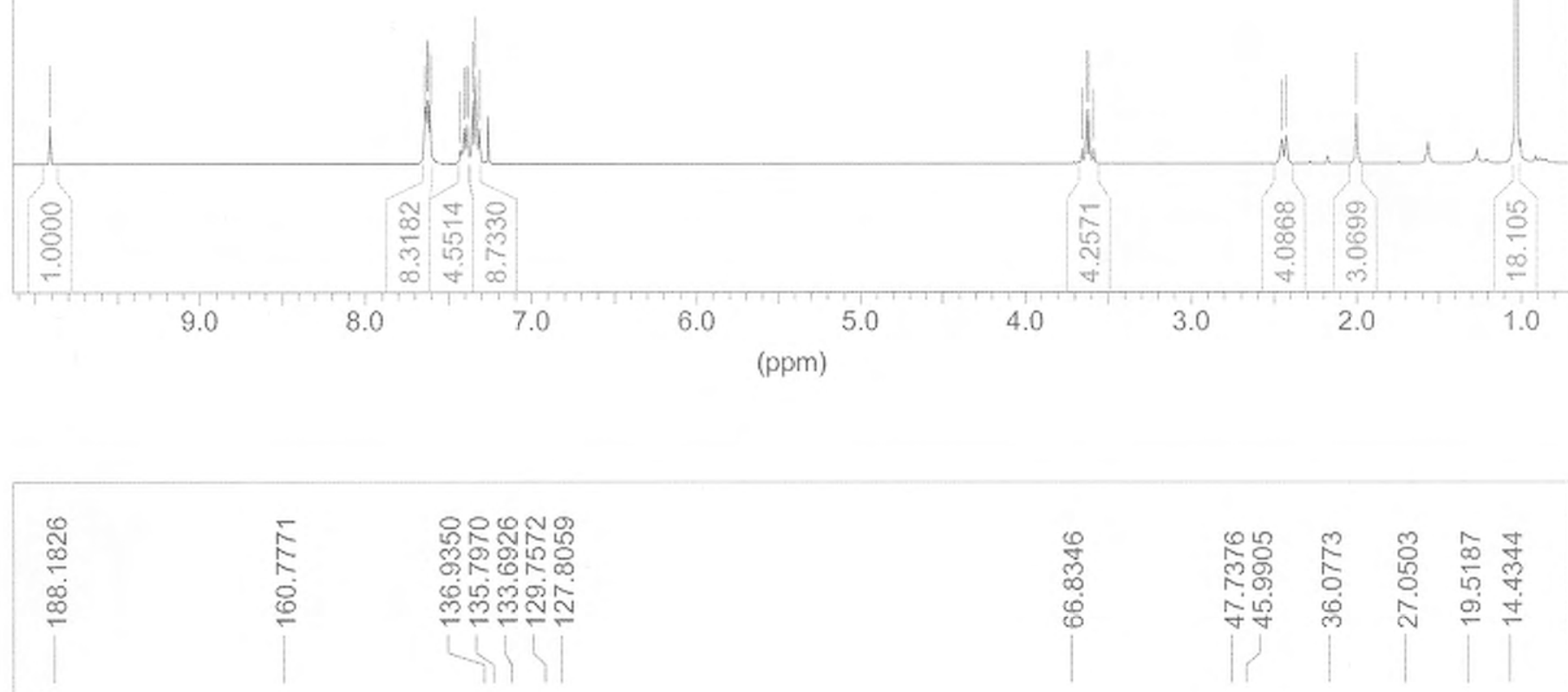


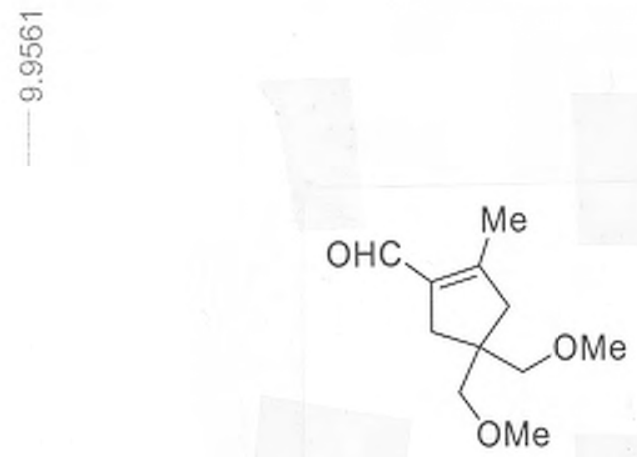

$3 c$

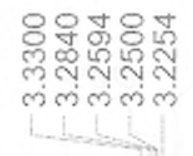

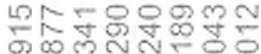

S以

งกัง vivin

Lar
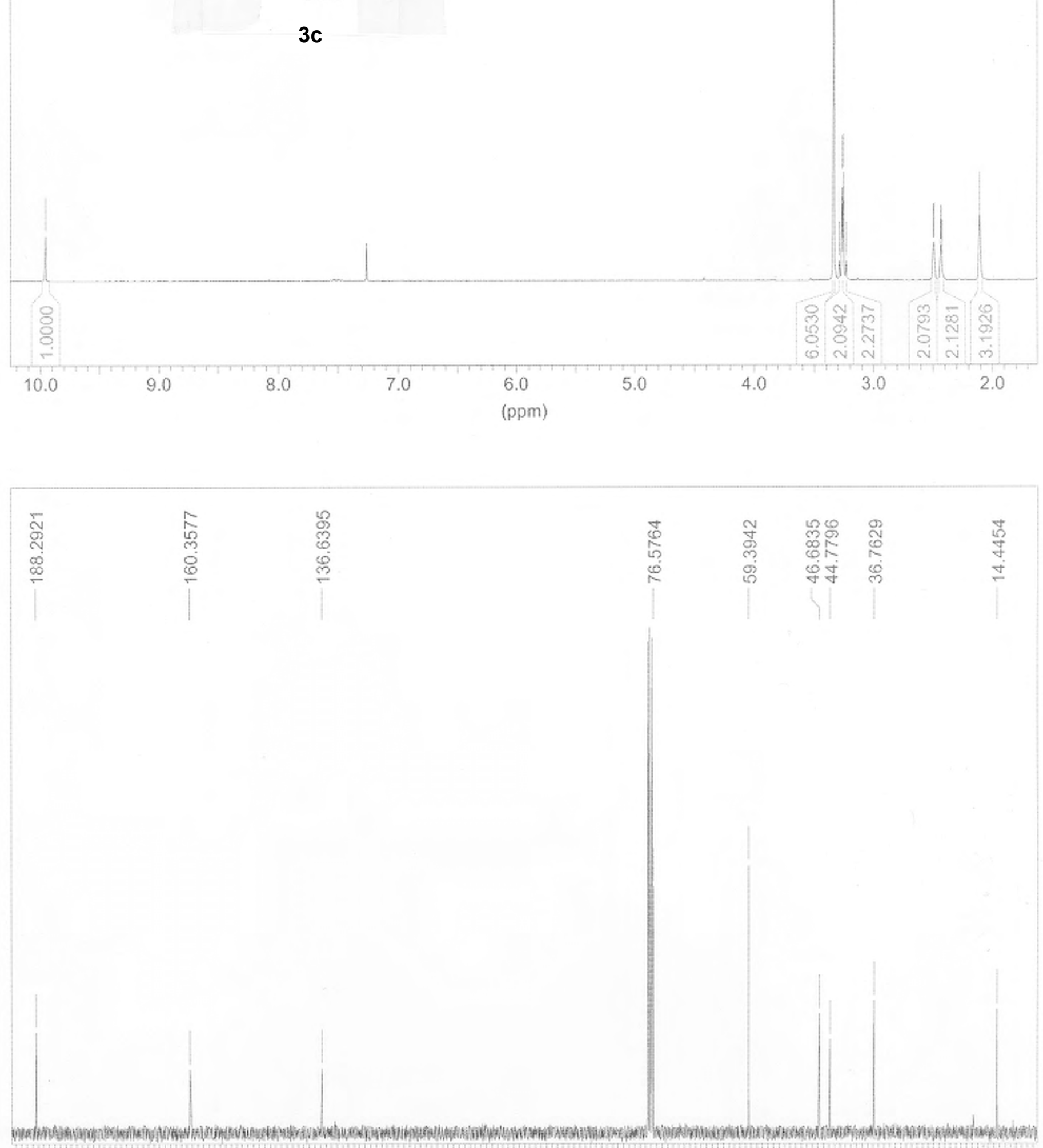
(ppm) 

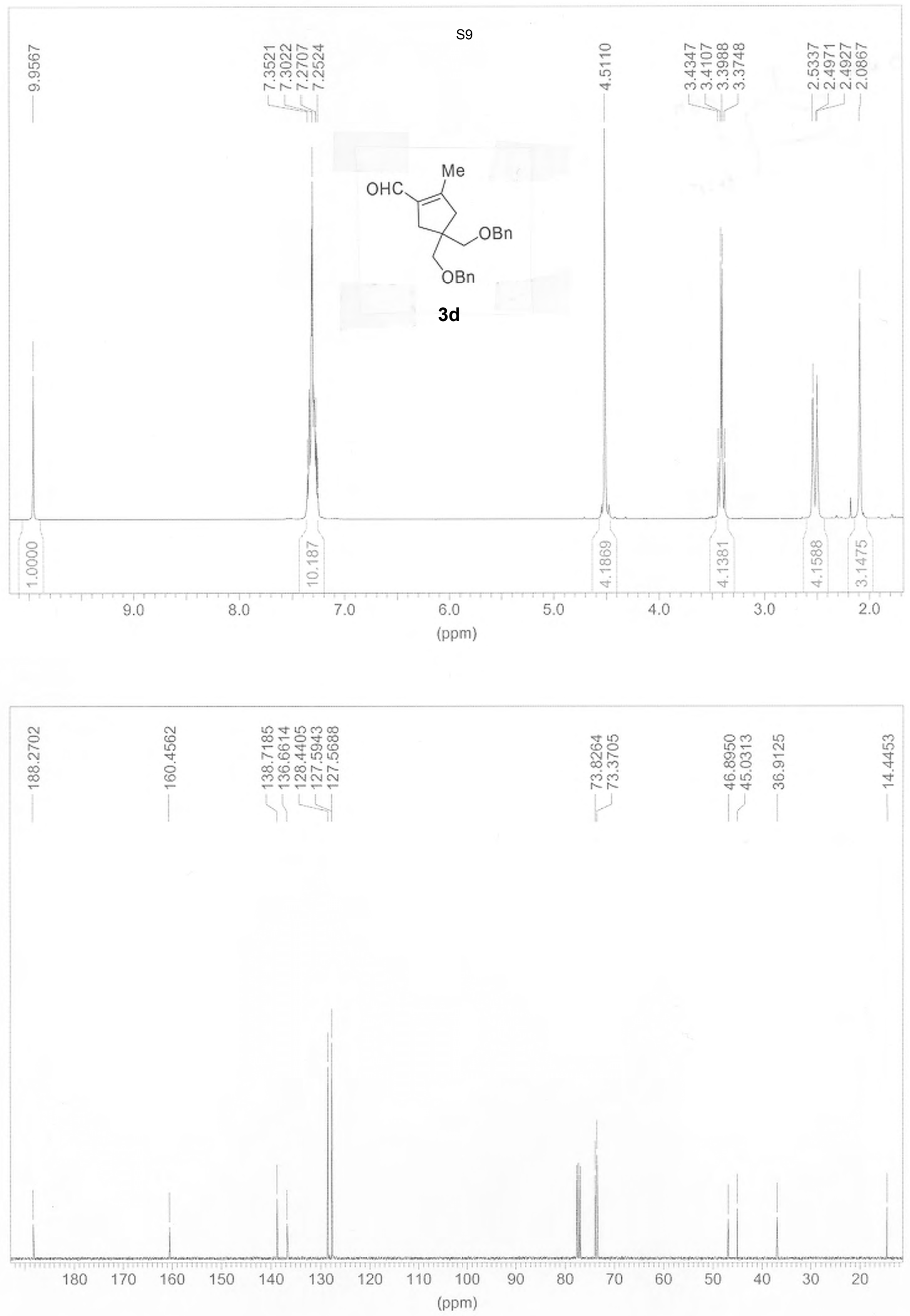


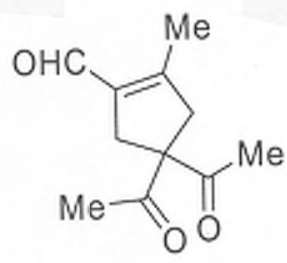

$3 e$

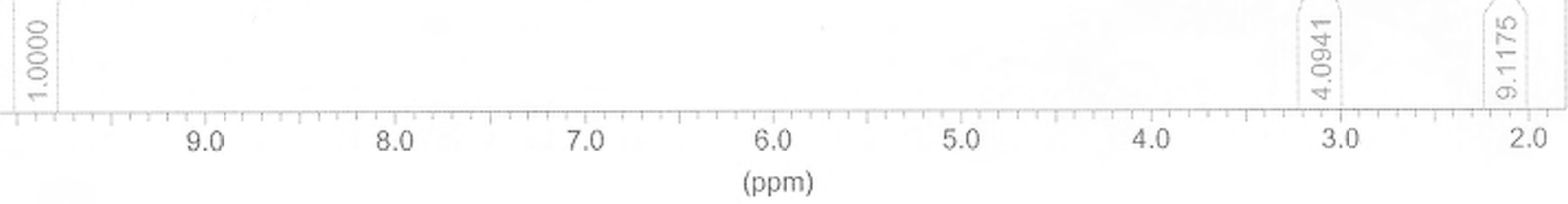

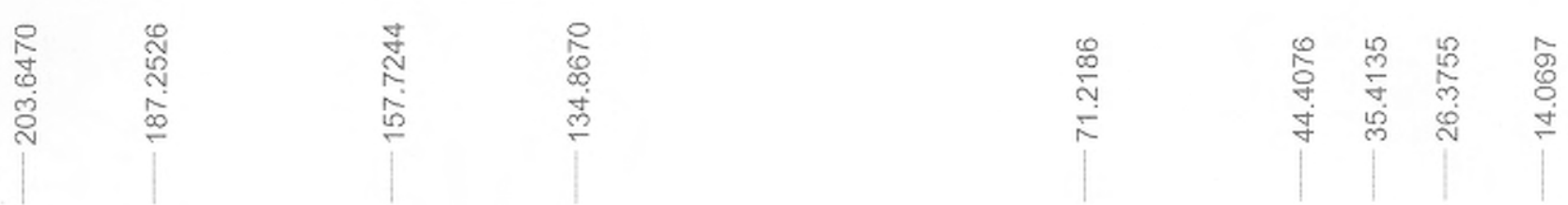




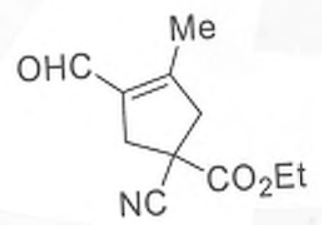

$3 f$

$$
\text { : }
$$

\section{(1)}
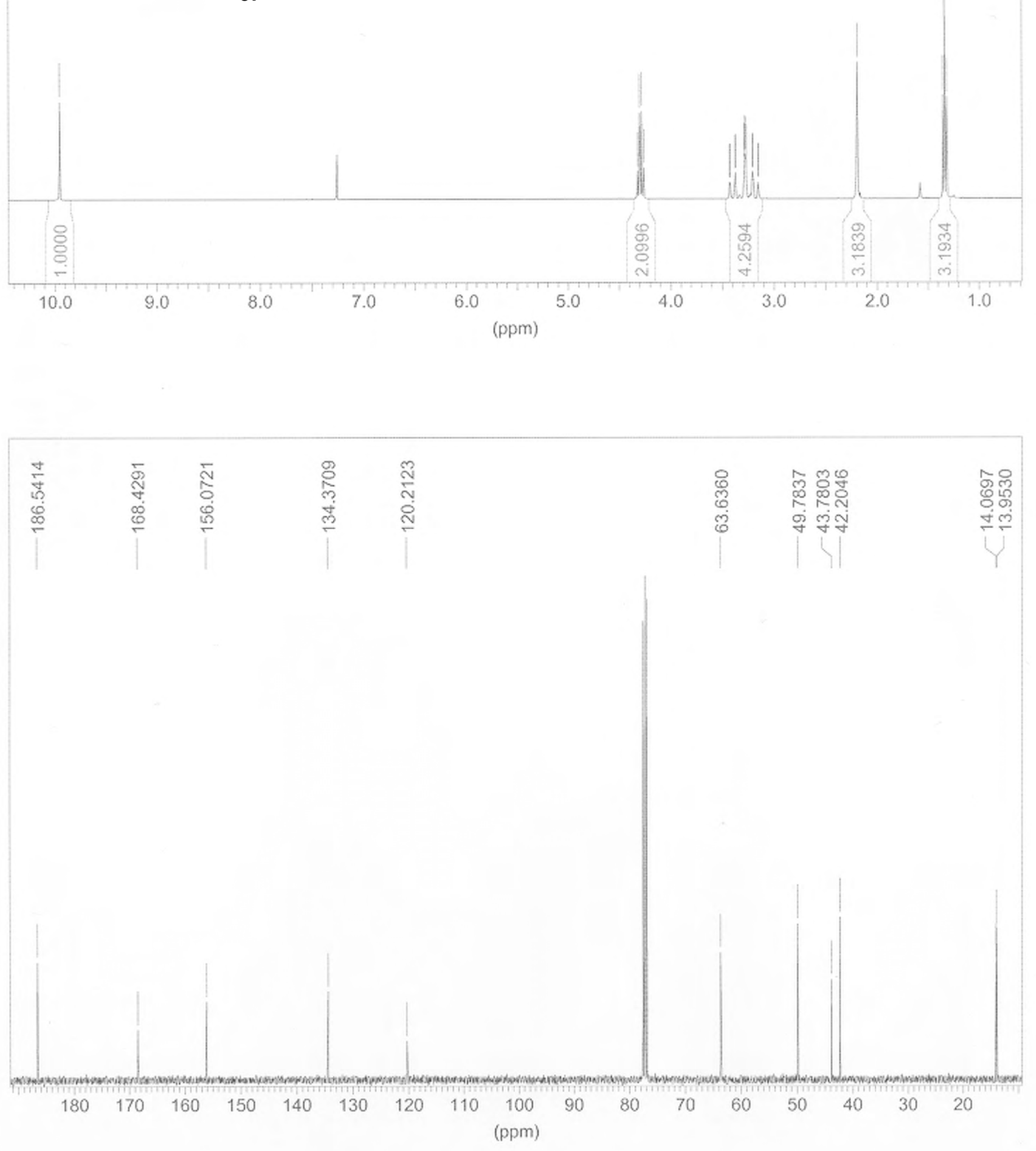
g)

0<smiles>CC1=C(C)c2ccccc2C1</smiles>

$3 g$
8

10.0

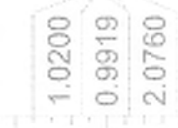

8.0

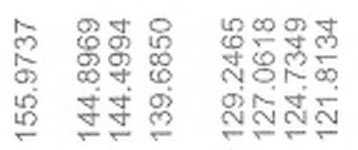

స్

ले

\section{0
0
0}




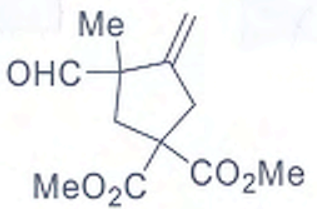

$2 \mathrm{~h}$
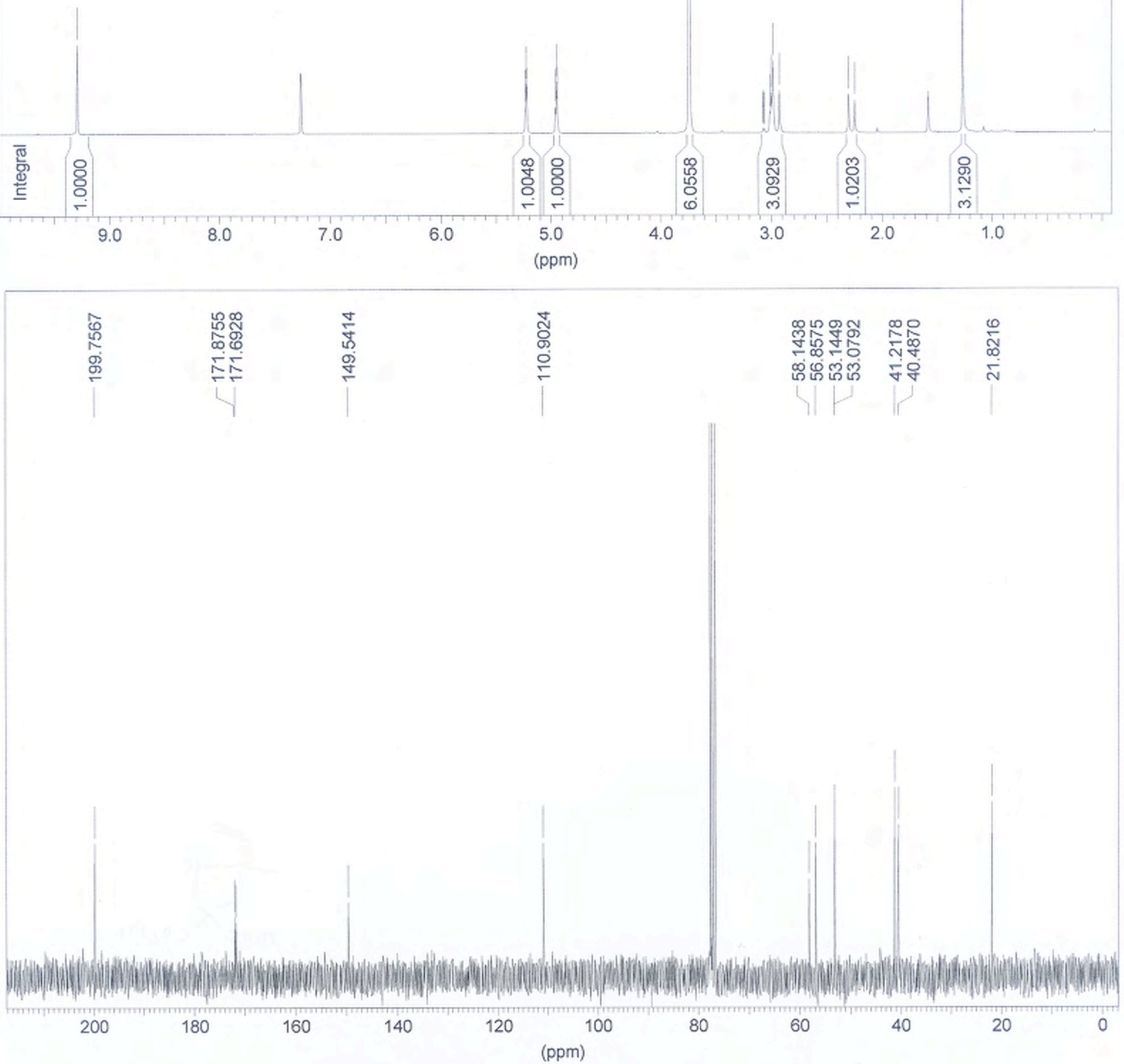


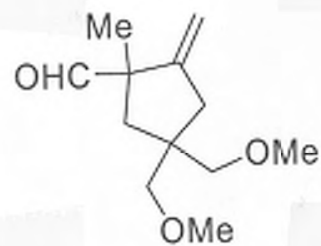

$2 \mathbf{i}$

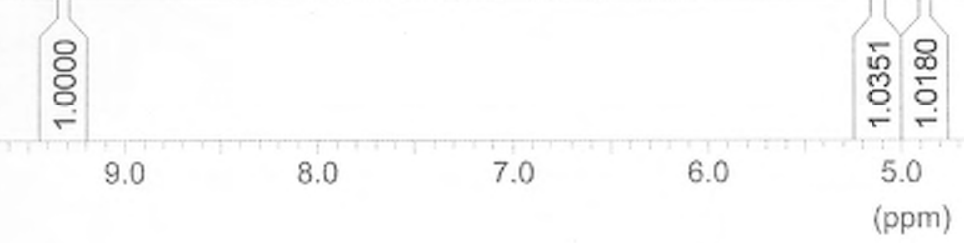

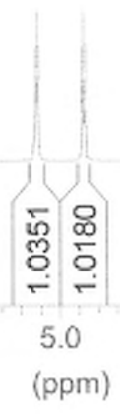

항

్․ำ
ஸู่

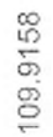

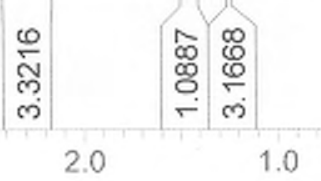

न 


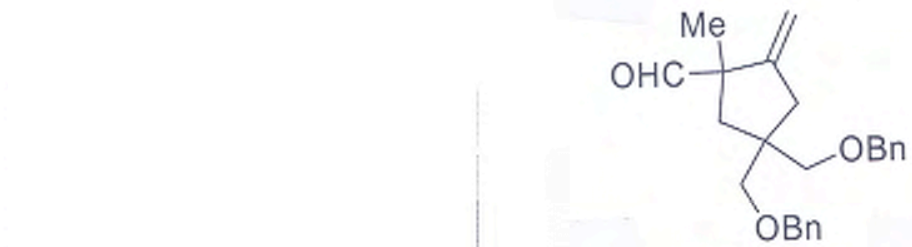

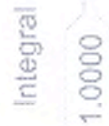

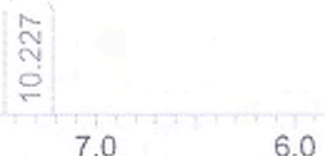

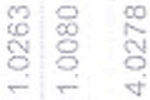
5.0
4.0
(ppm)

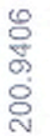

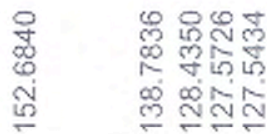

$\infty$
$\frac{1}{5}$
$\frac{15}{5}$

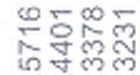

MMN

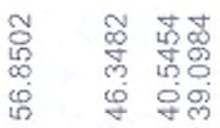

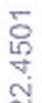

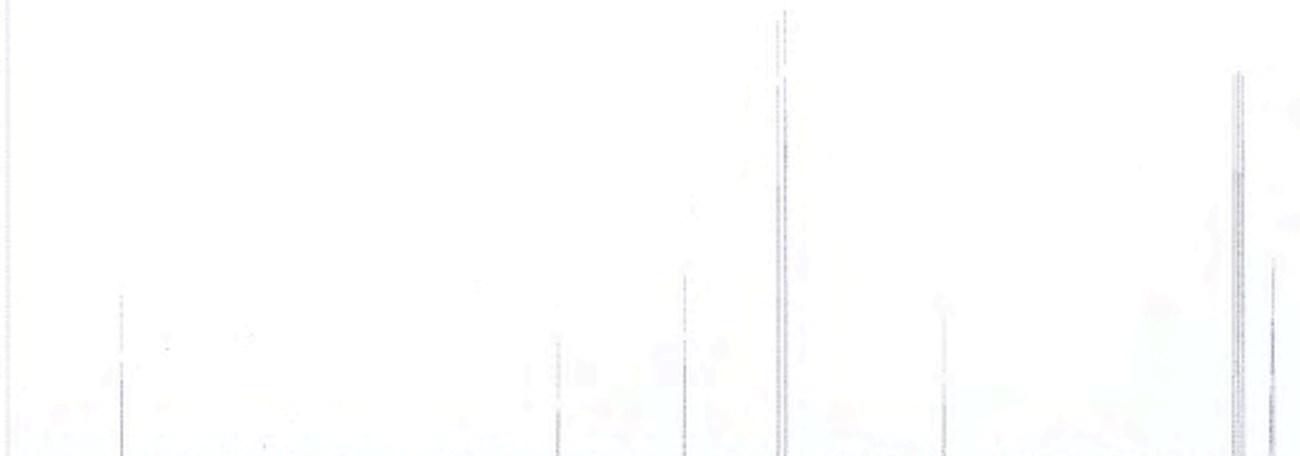

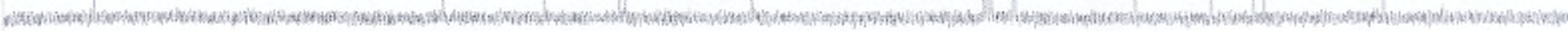




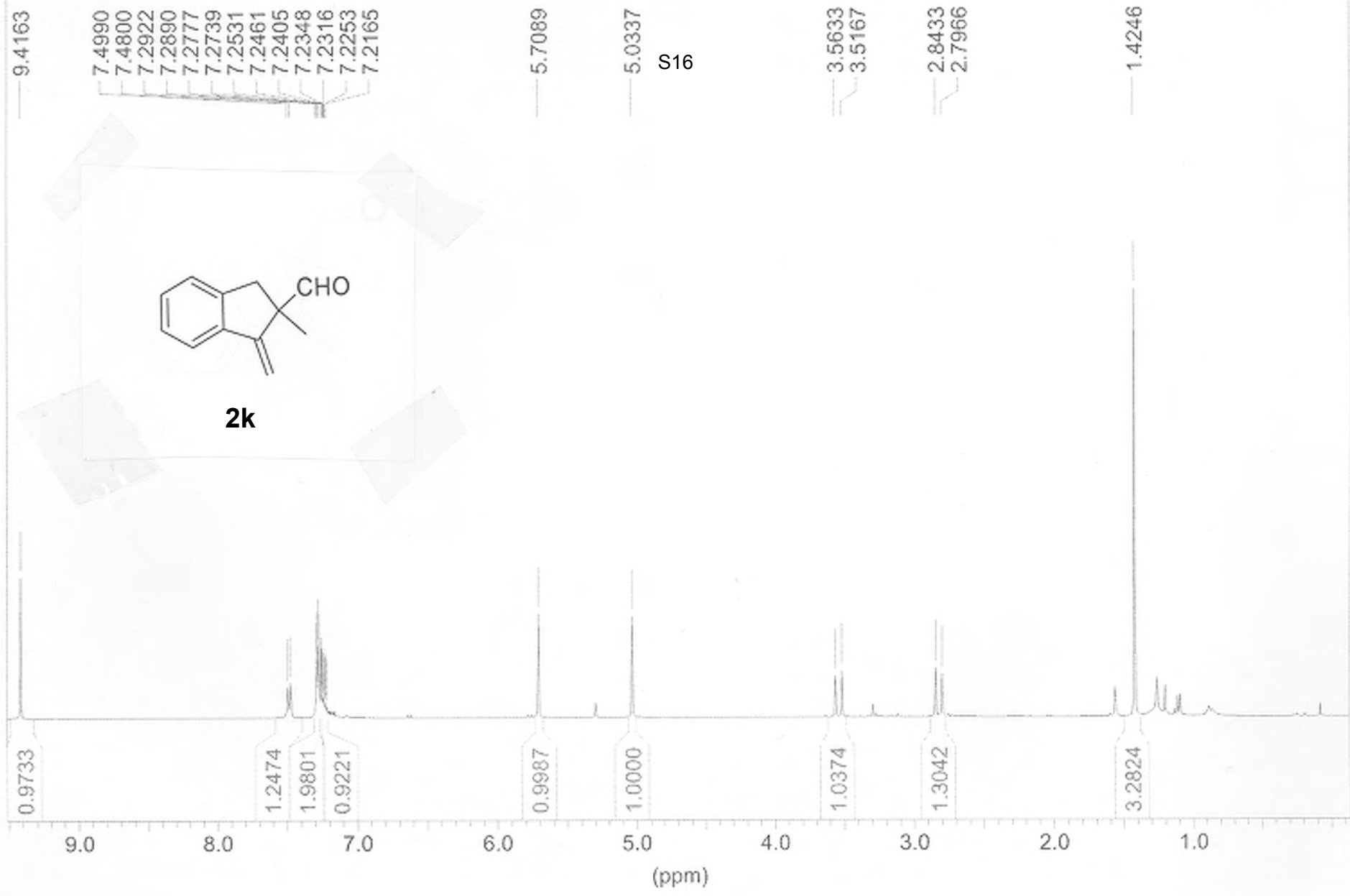

\begin{tabular}{|c|c|c|c|c|c|}
\hline 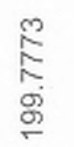 & 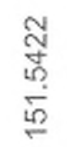 & 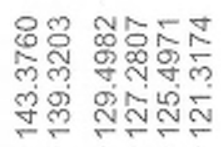 & $\frac{O}{\stackrel{+}{5}}$ & $\begin{array}{l}5 \\
0 \\
0 \\
0 \\
10\end{array}$ & 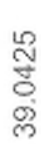 \\
\hline
\end{tabular}

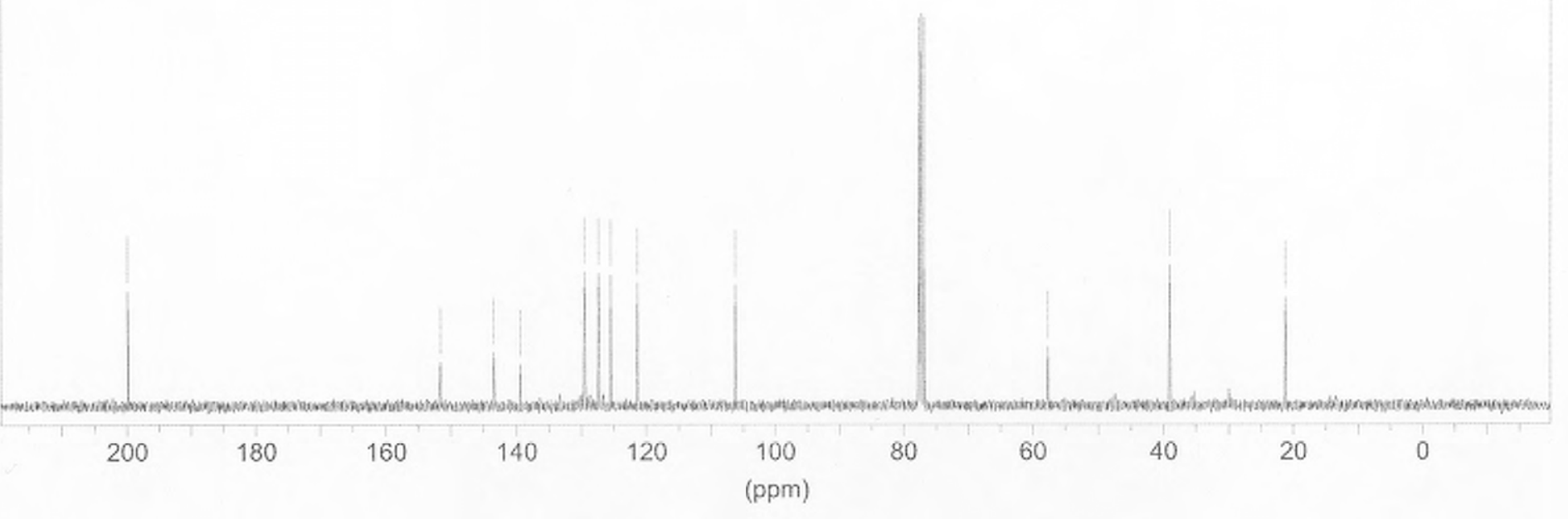




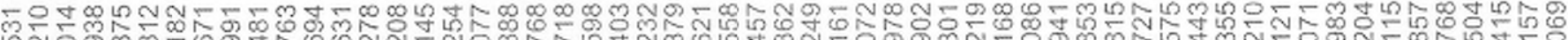

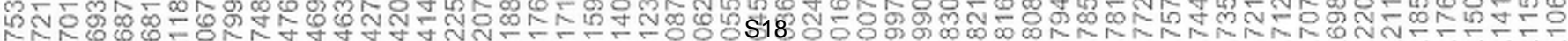
mmmm mm mand

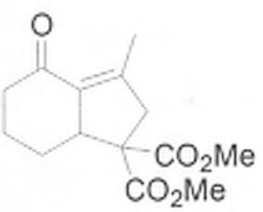

5

롤

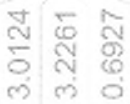

(ppm)

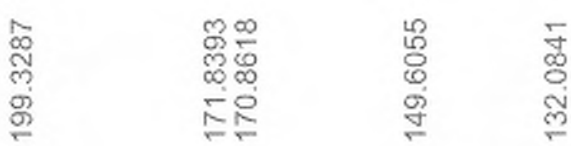

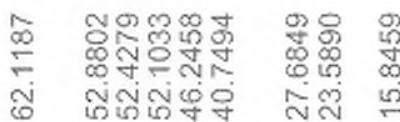




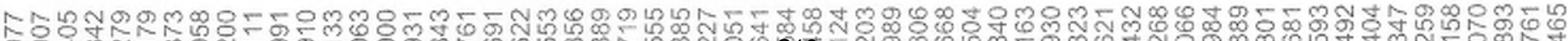

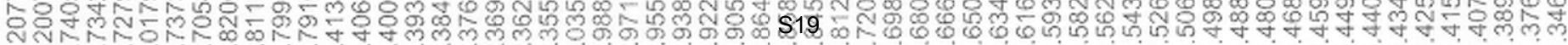

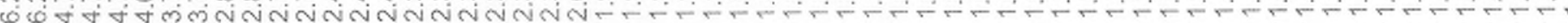

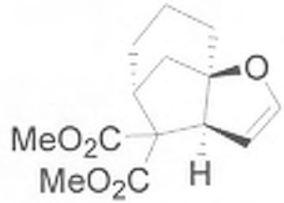

9

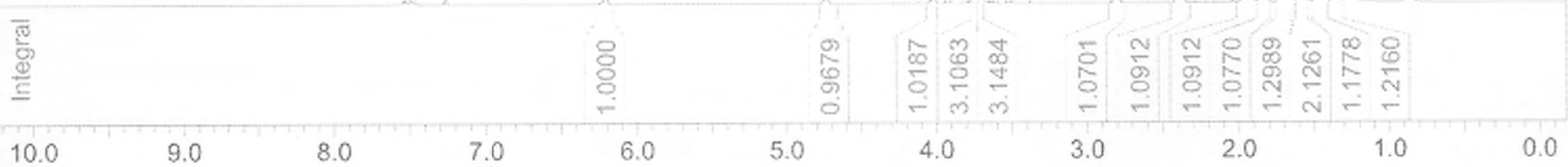

(ppm)

\begin{tabular}{|c|c|c|c|c|c|}
\hline $\begin{array}{l}3 \\
28 \\
0 \\
0 \\
0 \\
0 \\
5\end{array}$ & 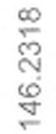 & 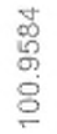 & $\begin{array}{l}\text { लె } \\
\text { ले } \\
\text { ले }\end{array}$ & $\begin{array}{l}\infty \\
\text { 号 } \\
\stackrel{8}{0}\end{array}$ & 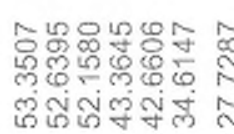 \\
\hline
\end{tabular}


${ }^{\star * \star}$ Current Data Parameters **

NAME

bciii79f1

EXPNO

11

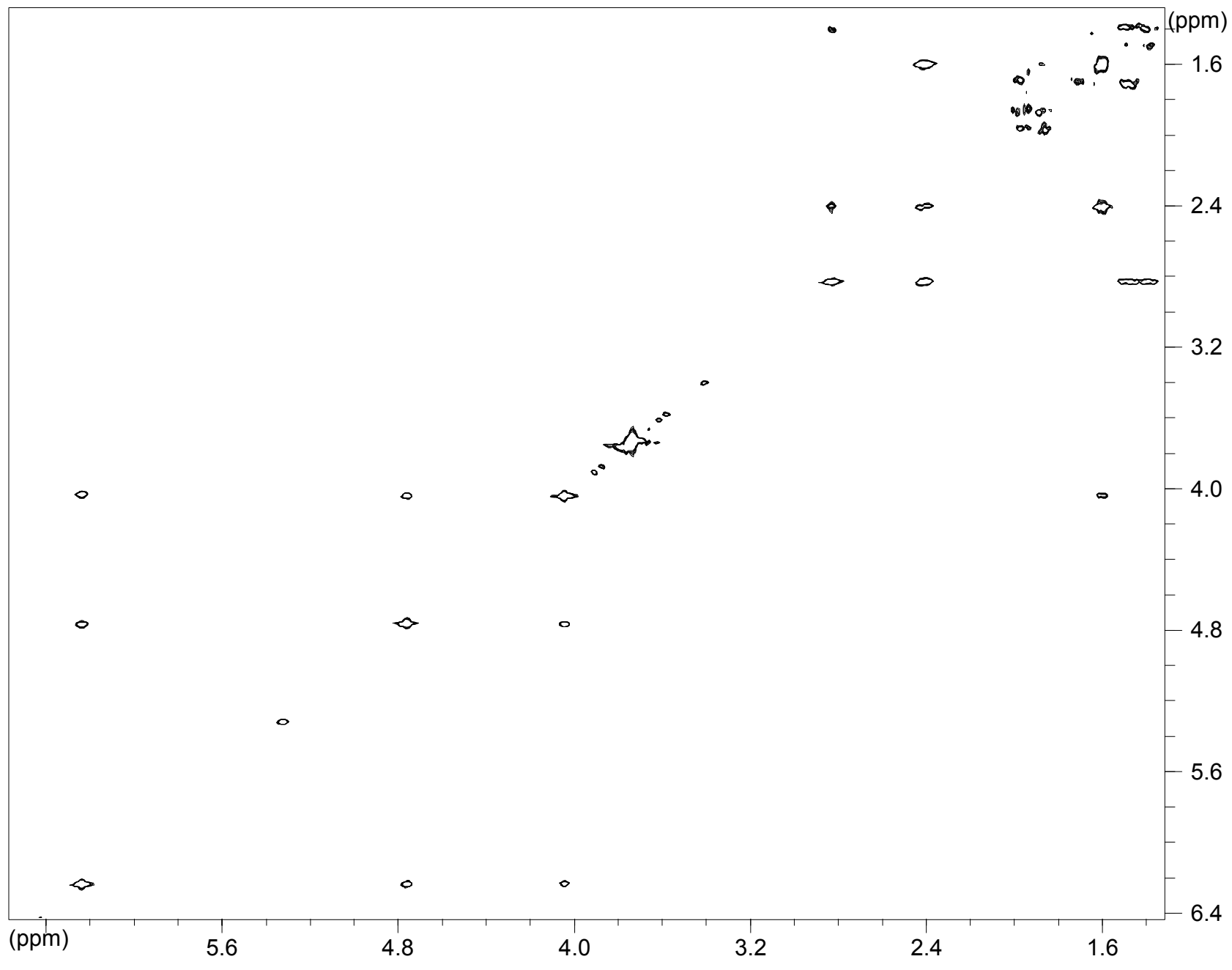

PROCNO : 


\section{Bruker}

${ }^{* *}$ Current Data Parameters ***

NAME

bciii79f1

EXPNO

13

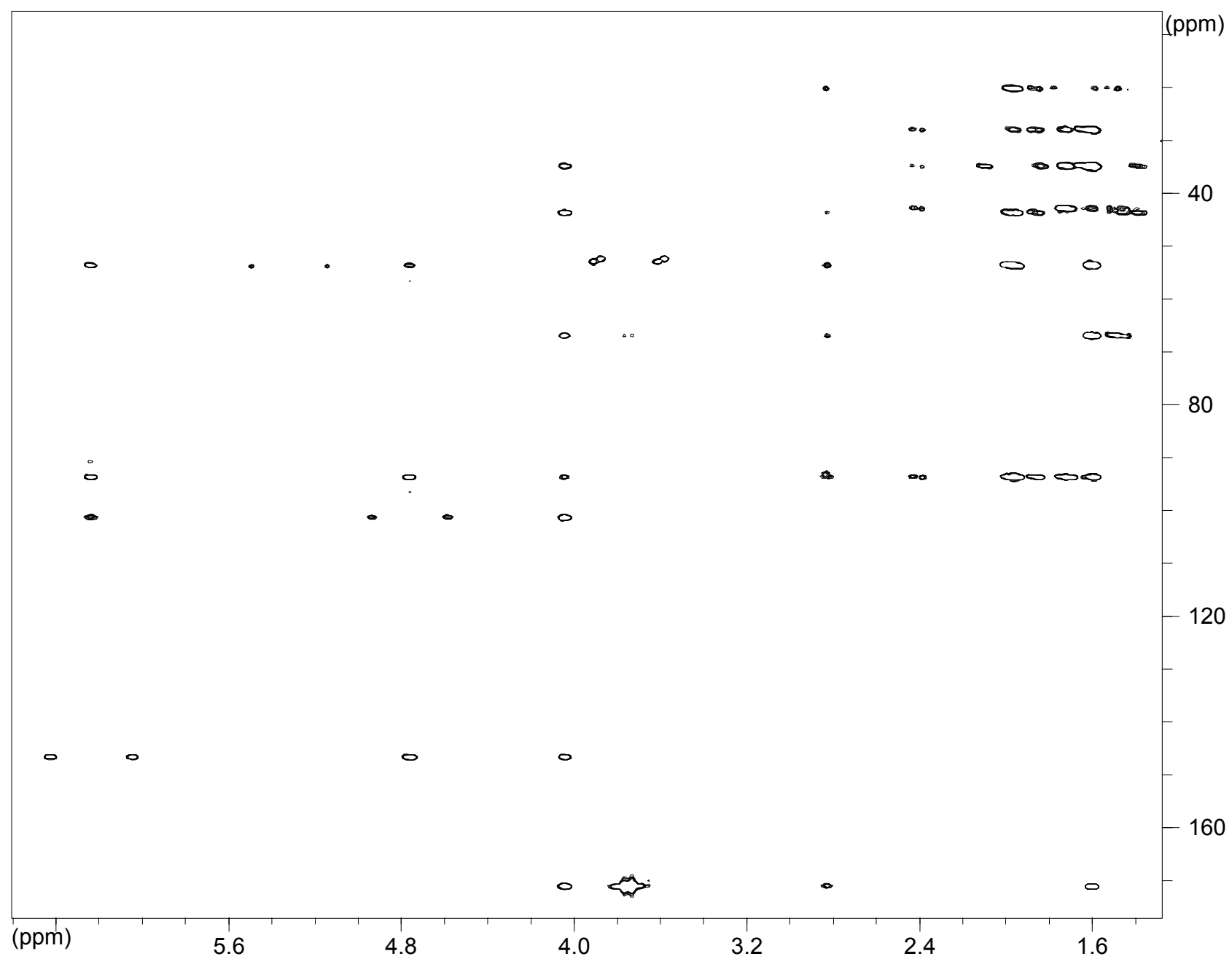

pm) 